\title{
Peripheral pulmonary nodules: Relationship between multi-slice spiral CT perfusion imaging and tumor angiogenesis and VEGF expression
}

\author{
Shu-Hua Ma*1,2, Hong-Bo Le ${ }^{1,2}$, Bao-hui Jia ${ }^{3}$, Zhao-Xin Wang ${ }^{1,2}$, Zhuang- \\ Wei Xiao ${ }^{1,2}$, Xiao-Ling Cheng1,2, Wei Mei ${ }^{1,2}$, Min Wu${ }^{1}$, Zhi-Guo $\mathrm{Hu}^{1,2}$ and $\mathrm{Yu}^{1}$ \\ Guang $\mathrm{Li}^{1}$
}

Address: ${ }^{1}$ Department of Radiology, First Affiliated Hospital, Medical College of Shantou University, Shantou 515041, PR China, ${ }^{2}$ Guangdong Key Laboratory of Medical Molecular Imaging, Shantou 515041, PR China and ${ }^{3}$ Guang Anmen Hospital, China Traditional Chinese Medical Sciences, Beijing 100053, PR China

Email: Shu-Hua Ma* - mashuhua6699@sina.com; Hong-Bo Le - hongbo1025@qq.com; Bao-hui Jia - myrrossee@yahoo.com.cn; ZhaoXin Wang - wzx425@gmail.com; Zhuang-Wei Xiao - xiaozhuangweiyxy@sina.com; Xiao-Ling Cheng - chengxiaoling0208@126.com; Wei Mei - meiweist@126.com; Min Wu - jwumin@yahoo.com; Zhi-Guo Hu - maxnow_hu@126.com; Yu-Guang Li - liyuguangyxy@sina.com

* Corresponding author

Published: 30 June 2008

BMC Cancer 2008, 8:186 doi:10.1186/147|-2407-8-186
Received: 27 November 2007

Accepted: 30 June 2008

This article is available from: http://www.biomedcentral.com/I47I-2407/8//86

(C) 2008 Ma et al; licensee BioMed Central Ltd.

This is an Open Access article distributed under the terms of the Creative Commons Attribution License (http://creativecommons.org/licenses/by/2.0), which permits unrestricted use, distribution, and reproduction in any medium, provided the original work is properly cited.

\begin{abstract}
Background: The aim of this study is to investigate the relationship between |6-slice spiral CT perfusion imaging and tumor angiogenesis and VEGF (vascular endothelial growth factor) expression in patients with benign and malignant pulmonary nodules, and differential diagnosis between benign and malignant pulmonary nodules.
\end{abstract}

Methods: Sixty-four patients with benign and malignant pulmonary nodules underwent 16-slice spiral CT perfusion imaging. The CT perfusion imaging was analyzed for TDC (time density curve), perfusion parametric maps, and the respective perfusion parameters. Immunohistochemical findings of MVD (microvessel density) measurement and VEGF expression was evaluated.

Results: The shape of the TDC of peripheral lung cancer was similar to those of inflammatory nodule. PH (peak height), PHpm/PHa (peak height ratio of pulmonary nodule to aorta), BF (blood flow), BV (blood volume) value of peripheral lung cancer and inflammatory nodule were not statistically significant (all $\mathrm{P}>0.05$ ). Both showed significantly higher $\mathrm{PH}, \mathrm{PH}$ pm/PHa, $\mathrm{BF}, \mathrm{BV}$ value than those of benign nodule (all $\mathrm{P}<0.05$ ). Peripheral lung cancer showed significantly higher PS (permeability surface) value than that of inflammatory nodule and benign nodule (all $P<0.05$ ). BV, $\mathrm{BF}, \mathrm{PS}, \mathrm{MTT}, \mathrm{PH}, \mathrm{PH} \mathrm{pm} / \mathrm{PHa}$, and MVD among three groups of peripheral lung cancers were not significantly (all $\mathrm{P}>0.05$ ). In the case of adenocarcinoma, $\mathrm{BV}, \mathrm{BF}, \mathrm{PS}, \mathrm{PHpm} / \mathrm{PHa}$, and MVD between poorly and well differentiation and between poorly and moderately differentiation were statistically significant (all $P<0.05$ ). The peripheral lung cancers with VEGF positive expression showed significantly higher $\mathrm{PH}, \mathrm{PHpm} / \mathrm{PHa}, \mathrm{BF}, \mathrm{BV}, \mathrm{PS}$, and MVD value than those of the peripheral lung cancer with VEGF negative expression, and than those of benign nodule with VEGF positive expression (all $\mathrm{P}<0.05$ ). When investigating VEGF negative expression, it is found that $\mathrm{PH}, \mathrm{PHpm} /$ $\mathrm{PHa}$, and MVD of inflammatory nodule were significantly higher than those of peripheral lung cancer, PS of inflammatory nodule were significantly lower than that of peripheral lung cancer (all 
$\mathrm{P}<0.05)$. $\mathrm{PH}, \mathrm{PH}$ pm/PHa, BF, and $\mathrm{BV}$ of benign nodule were significantly lower than those of inflammatory nodule (all $\mathrm{P}<0.05)$, rather than PS and MTT (mean transit time) (all $\mathrm{P}>0.05)$. $\mathrm{PH}$, $\mathrm{PHpm} / \mathrm{PHa}, \mathrm{BV}$, and PS of benign nodule were significantly lower than those of peripheral lung cancer (all $P<0.05$ ). In the case of VEGF positive expression, MVD was positively correlated with $\mathrm{PH}, \mathrm{PHpm} / \mathrm{PHa}, \mathrm{BF}, \mathrm{BV}$, and PS of peripheral lung cancer and PS of benign nodule (all $\mathrm{P}<0.05$ ).

Conclusion: Multi-slice spiral CT perfusion imaging closely correlated with tumor angiogenesis and reflected MVD measurement and VEGF expression. It provided not only a non-invasive method of quantitative assessment for blood flow patterns of peripheral pulmonary nodules but also an applicable diagnostic method for peripheral pulmonary nodules.

\section{Background}

Pulmonary nodule is a kind of common disease found in the lung. Sometimes it is difficult to directly identify between malignancy and benignancy of peripheral pulmonary nodule by using morphology features because of changes in the blood vessel volume, perfusion and capillary permeability in new tumor vessels which may result in the changes of blood patterns [1-3]. The method of perfusion imaging is effective to detect the differentiation of blood supply between benign and malignant pulmonary nodules in quantity and quality [4-8].

The purpose of this study was to quantitatively evaluate 16-slice spiral CT perfusion imaging to describe the blood patterns of benign and malignant pulmonary nodules and to investigate the relationship between CT perfusion imaging and tumor angiogenesis and vascular endothelial growth factor (VEGF) expression in patients with benign and malignant pulmonary nodules, and the differential diagnosis between benign and malignant pulmonary nodules by using perfusion imaging of 16-slice spiral CT.

\section{Methods \\ I. Patients}

From January 2005 to December 2006, 69 patients with peripheral pulmonary nodule were studied with informed consent. All the tumors were conformed by surgery. The patients underwent 16-slice spiral CT perfusion scanning in less than a week before surgery. No patients received any therapy (such as chemotherapy) before surgery. Five of 69 patients who could not hold their breath, led to errors in perfusion values and artifacts on parametric maps, and so were excluded. The other 64 patients (39 men and 25 women; age range, 26-75 years; mean age \pm $\mathrm{SD}, 51.27 \pm 13.83$ years; approximately spherical; longaxis diameters range, $1.6-4.2 \mathrm{~cm}$, mean diameters, 2.8 $\mathrm{cm}$ ) were included.

The 64 patients included 39 cases of peripheral lung cancer (24 adenocarcinoma, 11 squamous cell carcinoma, 2 large cell carcinoma, and 2 small cell carcinoma, long-axis diameters range, $1.7-4.2 \mathrm{~cm}$, mean diameters, $2.7 \mathrm{~cm}), 13$ cases of inflammatory nodules ( 8 active inflammatory granuloma and 5 pneumonia apostematosa, long-axis diameters range, $2.1-3.9 \mathrm{~cm}$, mean diameters, $3.2 \mathrm{~cm}), 12$ cases of benign nodules ( 9 tuberculoma and 3 hamartoma, long-axis diameters range, $1.6-3.0 \mathrm{~cm}$, mean diameters, $2.5 \mathrm{~cm}$ ). In the 39 cases of peripheral lung cancer, 24 adenocarcinoma (well, moderately, poorly differentiation are 9, 10, 5 cases respectively), 11 squamous cell carcinoma (well, moderately, and poorly differentiation are 6 , 4, 1 cases respectively), 11 large and small cell carcinoma (well, moderately, poorly differentiation are 6, 4, 1 cases respectively) (Table 1 ).

The experiments were performed with the approval of the human subjects ethics committee of the First Affiliated Hospital, Medical College of Shantou University.

\section{Computed tomography scanning}

Computed tomography perfusion imaging were performed by using a commercially available 16-slice spiral CT scanner (Lightspeed, GE, USA). The patients were instructed in breath-holding and in holding their breath at full suspended inspiration during quiet breathing so as to facilitate to repeat imaging of the same tissue volume.

The CT scan involved two steps. Firstly, a conventional CT scan was performed to find the tumor and determine the section of perfusion scan. The scan ranged from the thoracic inlet to the pulmonary base, with $1.25-\mathrm{mm}$ section thickness, 1.375 pitch, $10-\mathrm{mm}$ reconstruction thickness, $120-\mathrm{kV}$ tube voltage, 220-mA tube current. Secondly, CT perfusion scan was performed after the tumor was found.

Four adjacent sections containing the largest nodular diameter were selected as the target cross sections. Nonionic contrast medium ultravist 300 (300 mgl/ml)was

Table I: Differentiation of peripheral lung cancer

\begin{tabular}{lcccc}
\hline Differentiation of lung tumors & Cases & Well & Moderately & Poorly \\
\hline Adenocarcinoma & 24 & 9 & 10 & 5 \\
Squamous cell carcinoma & II & 6 & 4 & 1 \\
Large and small cell carcinoma & 4 & 3 & 0 & I
\end{tabular}


injected via a superficial antebrachial vein with pressure syringe at a flow rate of $4 \mathrm{ml} / \mathrm{s}$ and a total volume of 50 $\mathrm{ml}$. Perfusion scan was in a cine pattern, with $0.5 \mathrm{~s} /$ coil, 5 $\mathrm{mm}$ section thickness $\times 4,0$-mm interval, no inclination of scan frame, tube voltage of $120 \mathrm{kV}$, tube current of 60 $\mathrm{mA}$, exposition time of $40 \mathrm{~s}$, data acquisition time of $40 \mathrm{~s}$, delay time of $2 \mathrm{~s}$ (perfusion scan started after injection of contrast medium for $2 \mathrm{~s}$ ).

\section{Analysis of CT perfusion images}

The 316 images (79 images per section, 4 sections) with 5$\mathrm{mm}$ reconstruction thickness generated by CT perfusion were transmitted to AW4.1 workstation via a local area network. All the data were calculated quantitatively according to the calculation approach of the deconvolution model with the CT body perfusion software of GE perfusion 3 and produced perfusion parametric maps (assigned type). The analysis package offered a motion correction for in-plane movement. The four sections of data were respectively analyzed. Air and bone pixels are excluded from calculations by CT value (Hounsfield Unit, HU) thresholds, typically -120 to $240 \mathrm{HU}$.

Firstly, descending aorta (or innominate artery without descending aorta) in-plane of nodule was chosen as the input artery. A region of interest (ROI) with five pixels was hand drawn in the centre of the descending aorta. Secondly, three ROIs were hand drawn in every section of the nodular area of perfusion parametric maps; every ROI was about 70 pixels; and calcified, cystic, or necrotic areas were avoided as far as possible. The edges of the nodule were avoided to prevent partial volume. We examined an ROI that covered about two-thirds of the diameter of the nodule at the equator if the nodule was smaller.

The time density curves (TDC) of the descending aorta and pulmonary nodule ROI, peak height $(\mathrm{PH})$, peak height ratio of pulmonary nodule to aorta (PHpm/PHa), each type of perfusion parametric maps [blood flow (BF), blood volume (BV), mean transit time (MTT), permeability surface (PS)], and respective perfusion parameters for all four anatomic section locations available for each patient were calculated. Representative parameter values of three groups of pulmonary nodules were then averaged across the four sections.

Two radiologists experienced in chest CT respectively observed the TDCs and perfusion parametric maps.

\section{Immunohistochemical staining}

The position from which the pathologic tissue samples were drawn as far as possibe kept a parallel with the CT perfusion section. The tissue in each case was fixed by formalin and embedded by paraffin. Paraffin sections $(5 \mu \mathrm{m}$ thickness) were used for immunohistochemistry using a streptavidin peroxidase (SP) kit and a hematoxylin and eosin staining kit. The slices were deparaffinized and dehydrated in graded alcohols. Heat-induced antigen retrieval was performed by using a microwave oven and citrate buffer $(\mathrm{pH}, 6.0 ; 10 \mathrm{~mol} / \mathrm{L})$. All samples were immunostained by using the SP procedure with the monoclonal mouse antibodies VEGF (1:100 dilution) and the monoclonal rabbit antibodies CD34 (1:100 dilution) (both from Fuzhou Maixin Company, China). The positive breast cancer paraffin sections supplied with the SP kit were taken as positive controls and samples with primary antibody replaced by PBS were taken as negative controls.

VEGF positive brown staining is located in the cytoplasm. The VEGF staining was graded in terms of its extent and intensity shown by Mattern et al. [9]. To evaluate the VEGF expression, a score corresponding to the sum of both (a) staining intensity $(0=$ negative; $1=$ weak; $2=$ intermediate; $3=$ strong) and (b) percentage of positive cells $(0=0 \%$ positive cells; $1 \leq 25 \%$ positive cells; $2=25$ $50 \%$ positive cells; $3 \geq 50 \%$ positive cells) was established. The sum of (a) and (b) reached a maximum score of 6 . We observed 10 fields of vision by high time lens $(\times 400)$ and calculated the average score: $0-2$ is negative, more than 2 is positive, between 3 and 4 is weak positive expression, between 5 and 6 is strong positive expression.

The degree of angiogenesis was determined by means of MVD measurement in the defined areas of tissue sections according to the Weidner method [10]. Each slide was first scanned at low magnification $(\times 100)$ to determine five "hotspot" areas where the number of microvessels was at a maximum. Calcified or necrotic areas were excluded. MVD was counted in each of the five hotspot areas on a slide at high magnification $(\times 200)$. Any one brown staining endothelial cell or cell cluster that is obviously different from peripheral tissues and connective tissues was counted as a single vessel, and branch construct with discrete breaks was also counted as a single vessel. Appearance of erythrocyte and lumen of vessel may not be taken as calculation standard for MVD measurement; lumen of vessel that is more than eight erythrocytes in diameter and vessel that has thicker muscular layer were also not taken as calculation standard for MVD measurement.

A pathologist experienced in lung pathology recorded the histologic diagnosis of each pulmonary nodule. MVD measurement and VEGF expression were assessed by means of immunohistochemistry.

\section{Data and statistical analysis}

The pulmonary nodules were divided into three groups (peripheral lung cancer, inflammatory nodule, benign nodule). HU values of 11 time points $(2,6,10,14,18,22$, $26,30,34,38,42 \mathrm{~s}$ ) on each TDC of ROI at four anatomic 
Table 2: HU values showing I I time-points on the TDC of peripheral lung cancer, inflammatory nodule, and benign nodule

\begin{tabular}{|c|c|c|c|c|c|c|c|c|c|c|c|}
\hline Pathologic type & $2 S$ & $6 \mathrm{~S}$ & $10 \mathrm{~S}$ & $14 \mathrm{~S}$ & $18 \mathrm{~S}$ & $22 \mathrm{~S}$ & $26 \mathrm{~S}$ & $30 \mathrm{~S}$ & $34 \mathrm{~S}$ & $38 \mathrm{~S}$ & $42 \mathrm{~S}$ \\
\hline Peripheral lung & 11.46 & 21.66 & 29.94 & 36.05 & 40.76 & 48.86 & 51.77 & 50.16 & 45.81 & 43.55 & 42.16 \\
\hline Cancer & $\pm 4.4 \mathrm{I}$ & \pm 6.71 & \pm 7.99 & \pm 9.54 & \pm 10.64 & \pm 10.75 & \pm 10.21 & \pm 10.50 & \pm 9.67 & \pm 10.02 & \pm 9.54 \\
\hline Inflammatory & 18.55 & 35.60 & 40.86 & 43.96 & 50.60 & 56.97 & 63.64 & 60.57 & 49.52 & 43.98 & 42.02 \\
\hline Nodule & \pm 6.60 & \pm 7.59 & \pm 7.47 & \pm 8.05 & \pm 8.17 & \pm 6.37 & \pm 6.97 & \pm 8.11 & \pm 7.56 & \pm 8.41 & \pm 8.18 \\
\hline \multirow[t]{2}{*}{ Benign nodule } & 5.68 & 9.39 & 12.14 & 16.41 & 17.73 & 19.08 & 21.84 & 20.97 & 20.28 & 19.68 & 18.73 \\
\hline & \pm 5.25 & \pm 8.94 & \pm 8.82 & \pm 7.13 & \pm 5.83 & \pm 9.16 & \pm 13.28 & \pm 12.47 & \pm 10.84 & \pm 11.11 & \pm 10.46 \\
\hline
\end{tabular}

*Data is the mean $\pm S D$.

section locations available for each group of pulmonary nodules were recorded. Representative HU values of the respective time point were then averaged (Table 2). The TDCs of the three groups of pulmonary nodules were drawn by Graph software (OriginPro 7.5) (Figure 1).

The BV, BF, MTT, PS, PH, PHpm/PHa value of pulmonary nodule ROI for all four anatomic section locations available for each group of patients were recorded. Representative parameter values were then averaged. All statistical results were indicated with mean $\pm \mathrm{SD}$.

Chi-square test was used among two or three groups of number datas. P value was calculated by Exact Method.
One-way ANOVA was used to analyze statistical differences in two or three groups of measurement data, P value was calculated by LSD Method for equation of variance or by Tamhane Method for heterogeneity of variance. P values less than 0.05 were considered to indicate a statistically significant difference. Pearson coefficients were used to represent the relationship between the perfusion value (BV, BF, MTT, PS, PH, PHpm/PHa) and MVD measurement. All statistical analyses were performed by using a statistical software (SPSS 13.0 for Windows).

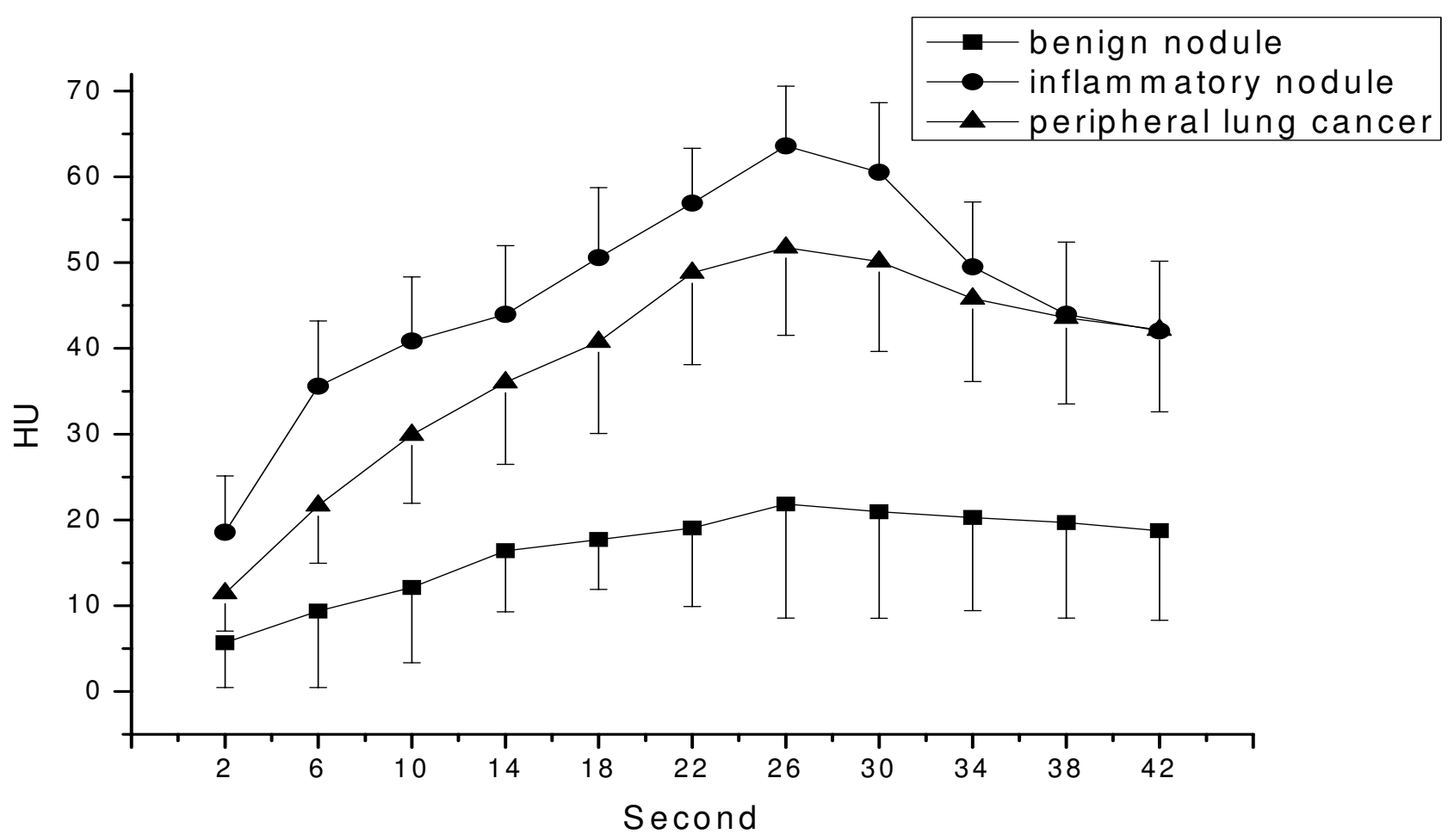

Figure I

Time density curve of peripheral lung cancer, inflammatory nodule, and benign nodule. 


\section{Results}

I. Time density curve of peripheral pulmonary nodule

The shape of the TDC of peripheral lung cancer was similar to that of inflammatory nodule. Both showed a steeper slope, and had an obviously increased nodular HU value. However, TDC of peripheral lung cancer changed little after reaching a peak. It became flat at the peak, and had a platform. However, TDC of inflammatory nodule declined immediately after reached peak and did not have a platform. Moreover, inflammatory nodule showed a higher peak than that of peripheral lung cancer. TDC of both was obviously different from that of benign nodule, in which, the TDC changed little, had no steeper slope, had a flat trend, and had no obvious increase in nodular HU value (Figures 1, 2A-6A).

\section{Immunohistochemistry}

In peripheral lung cancers, positive staining of CD34 were found in the tissues of small artery, small vein, capillary and blood vessel endothelium of lung cancer tissues, which were stained brown. Microvascular distributions were intense in the surrounding and interstitial substances of the nodules, and blood vessels were rare in essence and necrotic areas. Many immature tumor capillaries were found in the interstitial substance of the nodules. Many single or cluster endothelial cell clumps showed no lumina or their lumina were closed (Figures 2G-3G). In peripheral lung cancers, VEGF positive staining was located in the cytoplasm. The size of the cancer cell nucleus was bigger and atypia of the nucleus was obvious (Figures 2H, 3H). VEGF expression was found positive in 29 patients and negative in 10 patients.

In inflammatory nodules, positive staining of CD34 which displayed more expanded mature blood capillaries were observed (Figure. 4G). VEGF expression in all the 13 cases with inflammatory nodule were negative (Figure $4 \mathrm{H})$.

In benign nodules, positive staining of CD34 manifested that a smaller quantity of staining tumor capillaries were observed (Figures 5G, 6G). For VEGF expression, 3 patients were positive, 9 patients were negative (Figures $5 \mathrm{H}, 6 \mathrm{H})$.

MVD results of peripheral lung cancer, inflammatory nodule and benign nodule were $29.292 \pm 14.391,33.966 \pm$ 15.548 , and $7.959 \pm 2.328$, respectively. MVD values of peripheral lung cancer and inflammatory nodule were very high and not statistically significant $(P>.05)$. However, both MVD values were statistically significant higher than that of benign nodule (all $\mathrm{P}<.05$ ) (Tables 3, 4).

MVD among adenocarcinoma, squamous cell carcinoma, large and small cell carcinoma were not significantly (all
$\mathrm{P}>0.05$ ) (Table 5). In the case of adenocarcinoma, MVD between poorly and well differentiation and between poorly and moderately differentiation were statistically significant (all $\mathrm{P}<0.05$ ) (Tables 6, 7).

The peripheral lung cancers with VEGF positive expression showed significantly higher MVD value than that of peripheral lung cancers with VEGF negative expression ( $P$ $<.05)$. MVD of benign nodules with VEGF positive and negative expression were not statistically significant $(\mathrm{P}>$ .05) (Table 8).

For VEGF negative expression, MVD of inflammatory nodule were significantly higher than that of peripheral lung cancer $(\mathrm{P}<.05)$, MVD of benign nodule were significantly lower than that of peripheral lung cancer and inflammatory nodule $(\mathrm{P}<.05)$ (Tables 8,9$)$.

\section{Perfusion parameters of pulmonary nodules}

$\mathrm{PH}, \mathrm{PHpm} / \mathrm{PHa}, \mathrm{BF}$ and BV values of peripheral lung cancer and inflammatory nodule were not statistically significant (all $\mathrm{P}>.05$ ). Both showed significantly higher $\mathrm{PH}$, $\mathrm{PHpm} / \mathrm{PHa}, \mathrm{BF}, \mathrm{BV}$ value than those of benign nodule (all $\mathrm{P}<.05)$. MTT value was not statistically significant among the three groups of nodules (all $\mathrm{P}>.05$ ). Peripheral lung cancer showed significantly higher PS value than that of inflammatory nodule and benign nodule (all $\mathrm{P}<.05$ ). PS value of inflammatory nodule and benign nodule was not statistically significant $(\mathrm{P}>.05)$ (Tables 3,4$)$.

BV, BF, PS, MTT, PH, and PHpm/PHa among adenocarcinoma, squamous cell carcinoma, large and small cell carcinoma were not significantly (all $\mathrm{P}>0.05$ ) (Table 5). In the case of adenocarcinoma, BV, BF, PS, PHpm/PHa between poorly and well differentiation and between poorly and moderately differentiation were statistically significant (all $\mathrm{P}<0.05$ ) (Tables 6, 7).

The peripheral lung cancers with VEGF positive expression showed significantly higher $\mathrm{PH}, \mathrm{PHpm} / \mathrm{PHa}, \mathrm{BF}, \mathrm{BV}$, and PS values than those of the peripheral lung cancers with VEGF negative expression, and than those of benign nodule with VEGF positive expression (Table 8).

In the case of VEGF negative expression, the $\mathrm{PH}$, and $\mathrm{PHpm} / \mathrm{PHa}$ of inflammatory nodule were significantly higher than those of peripheral lung cancer (all $\mathrm{P}<.05)$. The PS of inflammatory nodule was significantly lower than that of peripheral lung cancer $(\mathrm{P}<.05)$. Both $\mathrm{BF}, \mathrm{BV}$, and MTT were not statistically significant (all P>.05). PH, $\mathrm{PHpm} / \mathrm{PHa}, \mathrm{BF}$, and BV of benign nodule were significantly lower than those of inflammatory nodule (all $\mathrm{P}<$ .05), rather than PS and MTT (all P > .05). PH, PHpm/ $\mathrm{PHa}, \mathrm{BV}$, and PS of benign nodule were significantly lower 


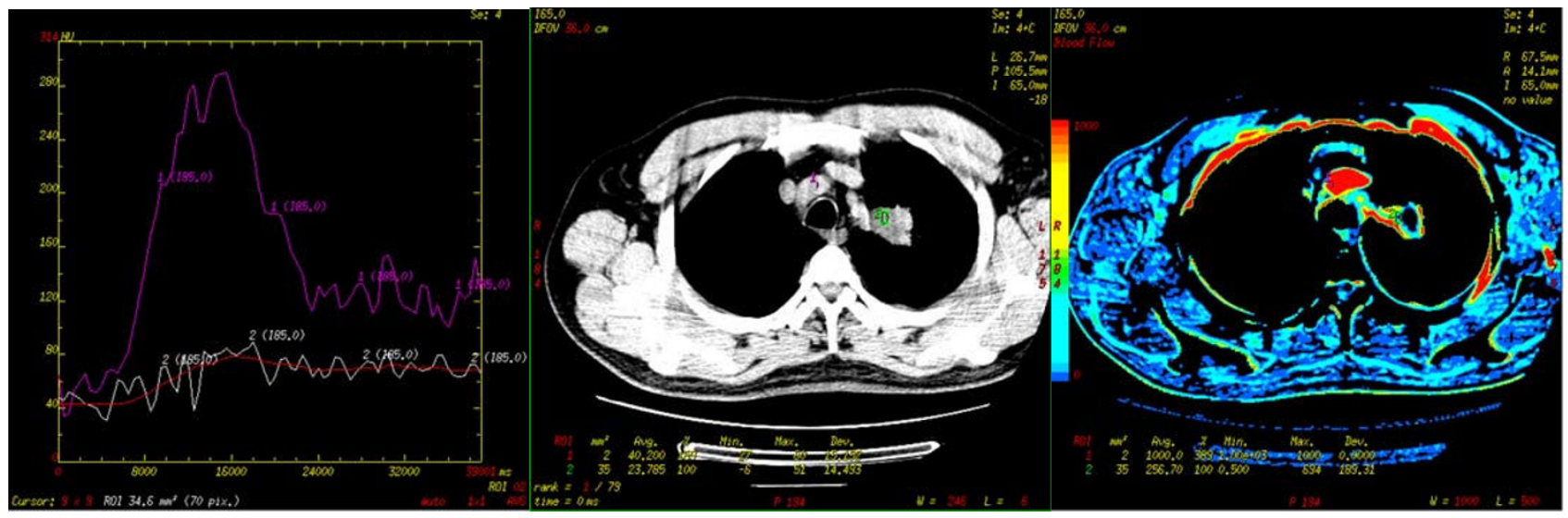

A

B

C

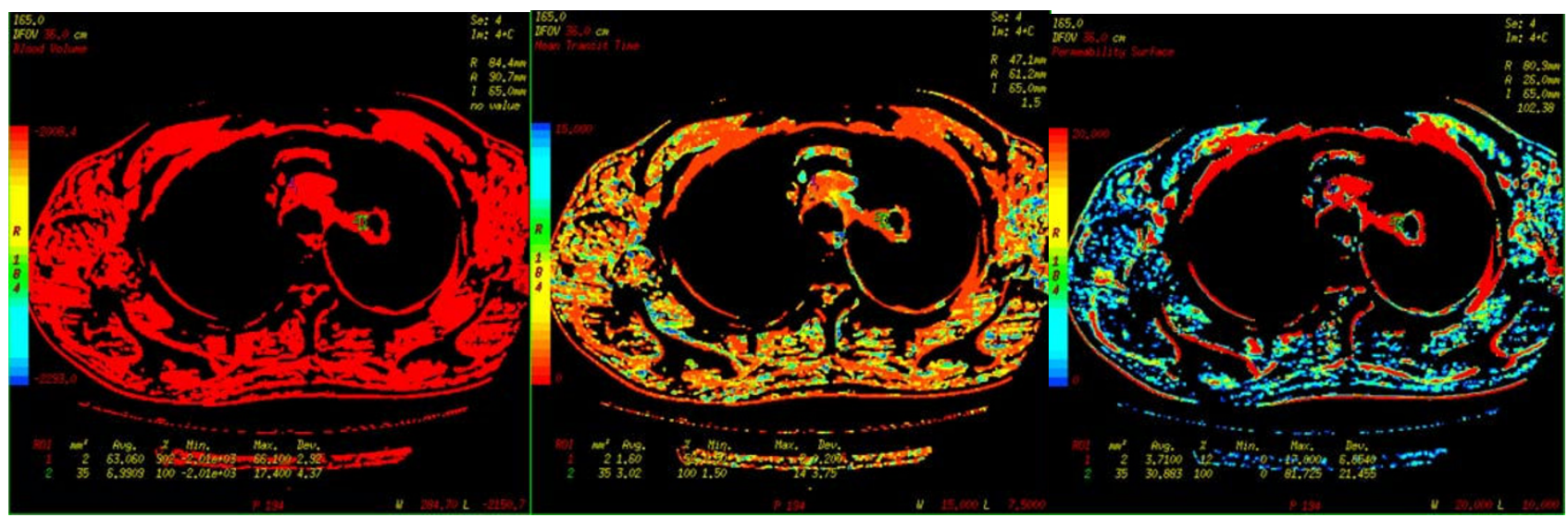

D

E

F

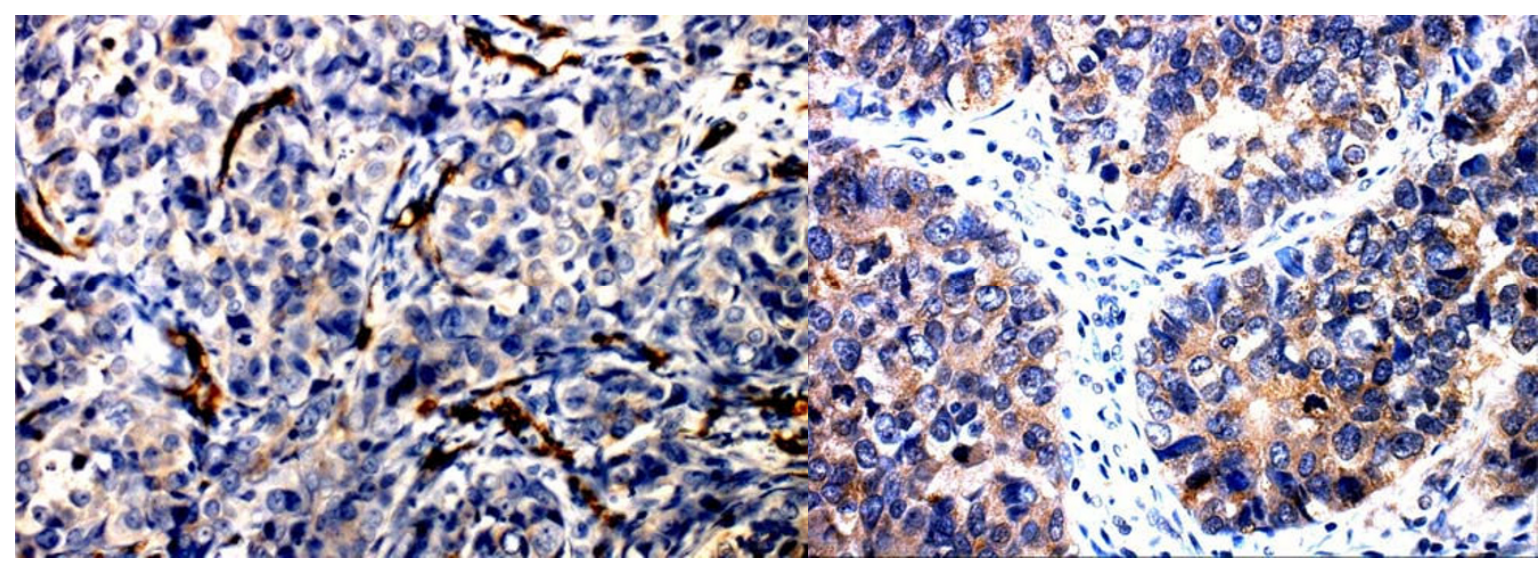

G

H

Figure 2

(A-H) Poorly differentiated adenocarcinoma found in the apicoposterior segment of superior lobe of the left lung of a 56 yearold male. (A) Time density curve. (B-F) (original image, BF, BV, MTT, PS) typel parametric maps, PS value is higher (30.883).

(G) CD34 staining shows many immature tumor microvessels $(\times 200)$. (H) VEGF expression is strong positive $(\times 400)$. 


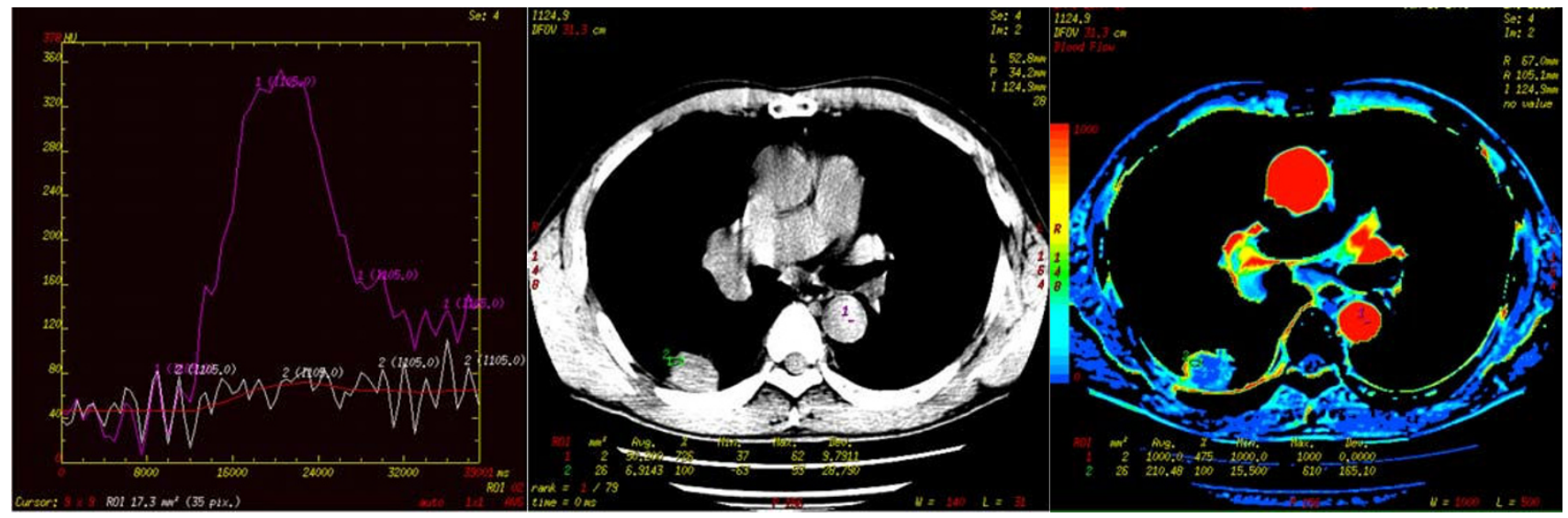

A

B

C

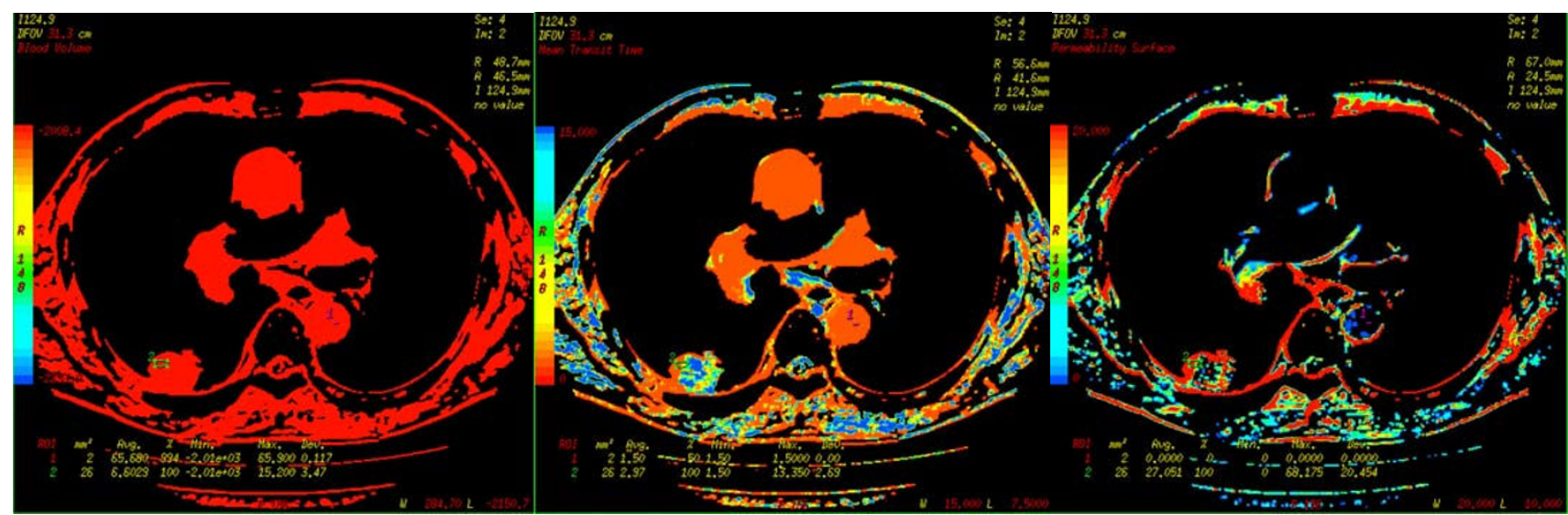

D

$\mathbf{E}$

$\mathbf{F}$



G

H

Figure 3

(A-H) Well differentiated squamous cell carcinoma found in the posterior basal segment of inferior lobe of the right lung of a 6l-year-old male. (A) Time density curve. (B-F) (original image, BF, BV, MTT, PS) Typell parametric maps, PS value is higher (27.05I). (G) CD34 staining shows many immature tumor microvessels $(\times 200)$. (H) VEGF expression is strong positive $(\times$ 400). 

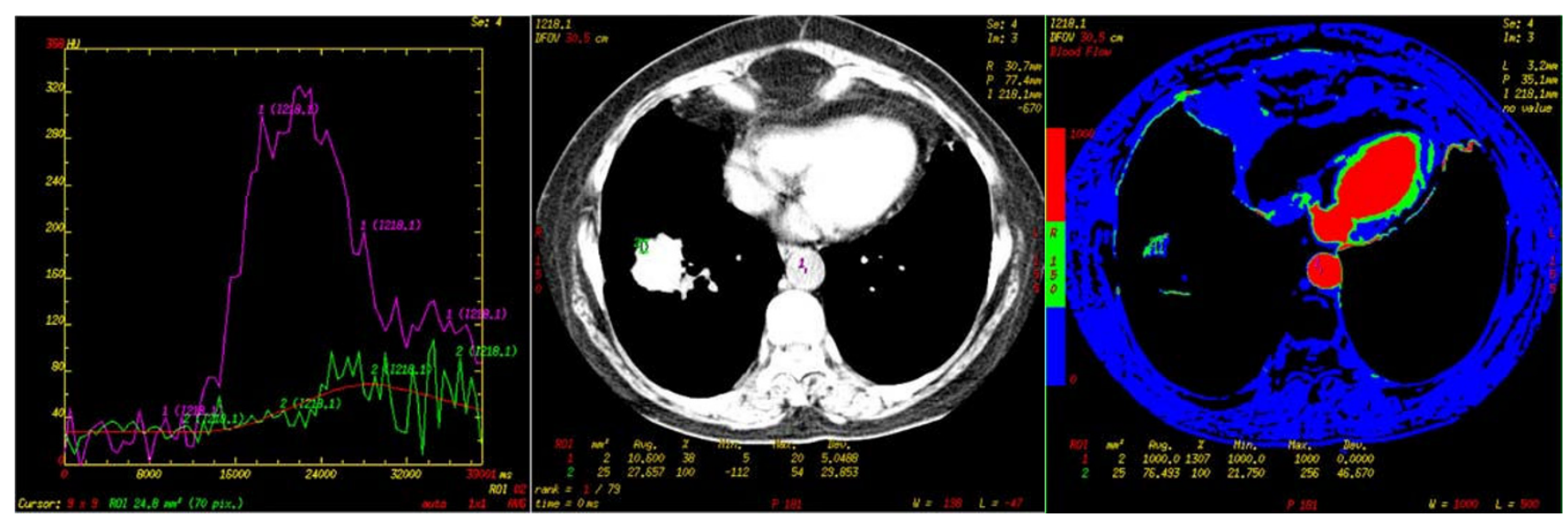

A

B

C



D

$\mathbf{E}$

F

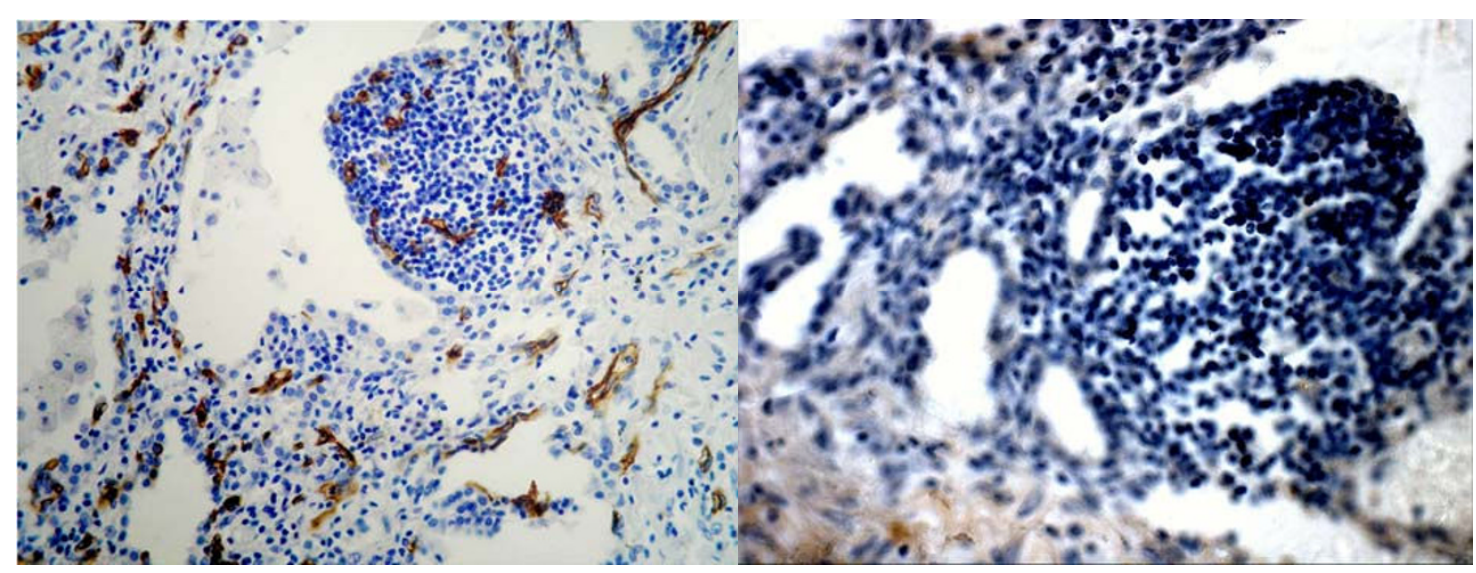

G

H

Figure 4

(A-H) Inflammatory nodule found in segmentum basale anterius of inferior lobe of the right lung of a 46-year-old male. (A) Time density curve. (B-F) (original image, BF, BV, MTT, PS) Typelll parametric maps, PS value was lower (5.63). (G) CD34 staining shows many expanded mature microvessels $(\times 200)$. (H) VEGF expression is negative $(\times 400)$. 




A

B

C

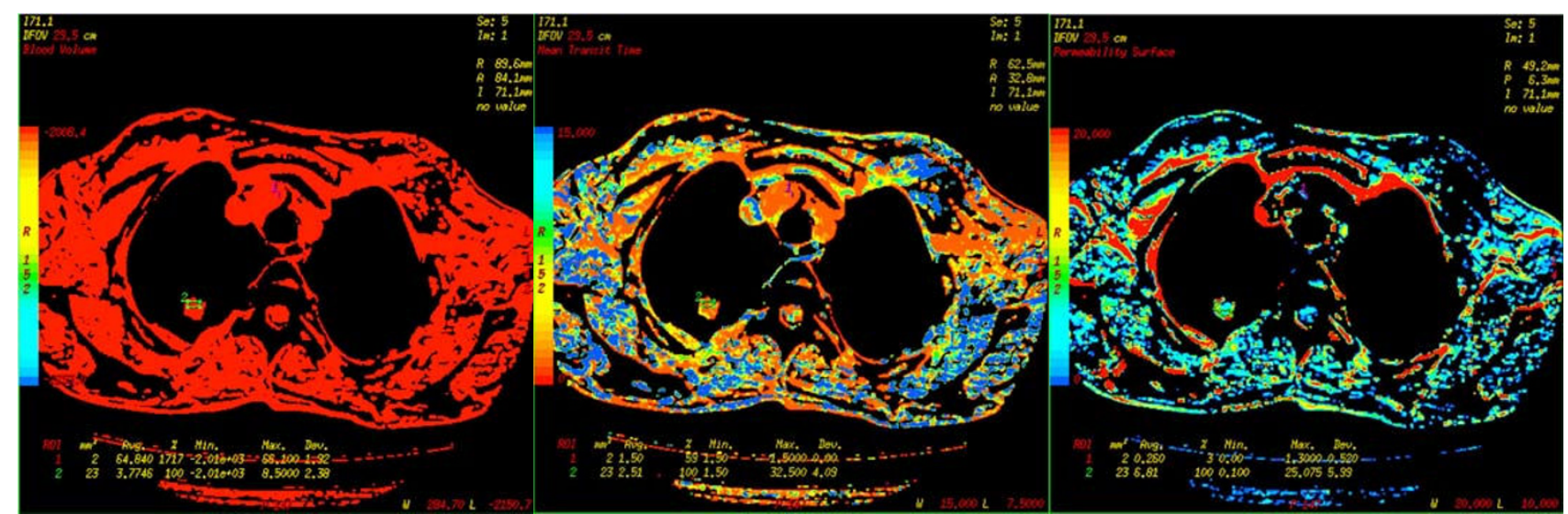

D

$\mathbf{E}$

$\mathbf{F}$

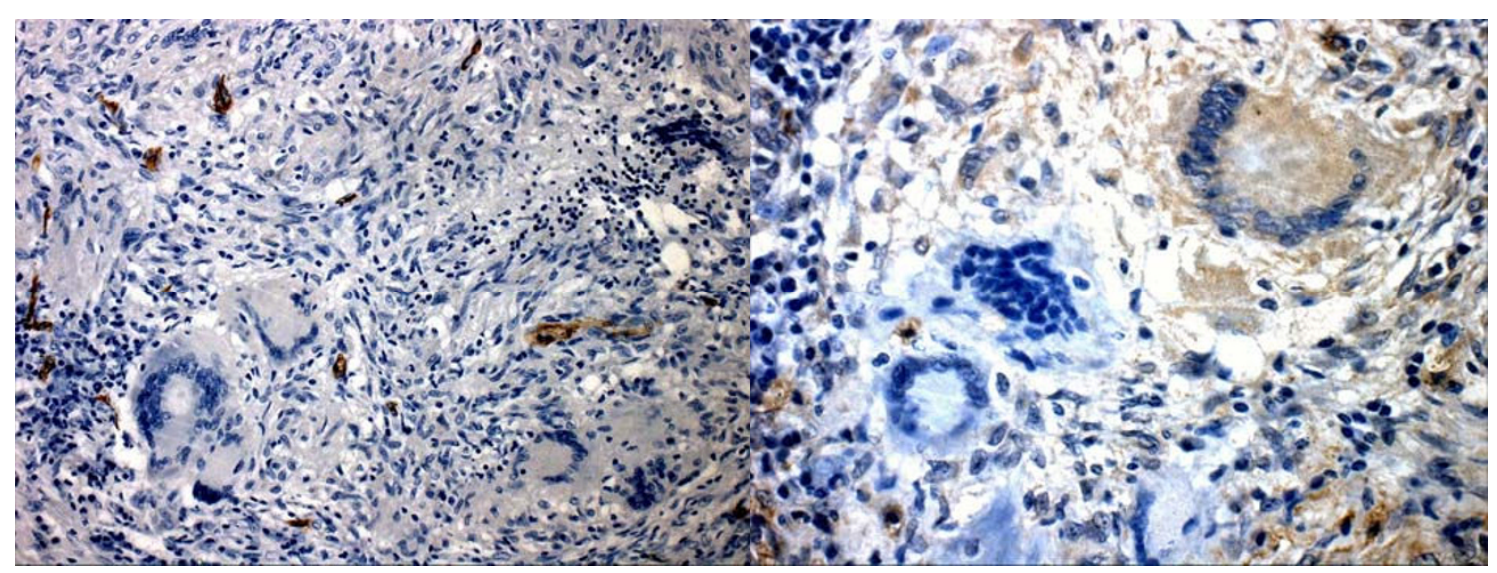

G

H

Figure 5

(A-H) Tuberculoma found in apical segment of superior lobe of the right lung of a 39-year-old female. (A) Time density curve. (B-F) (original image, BF, BV, MTT, PS) typel parametric maps, PS value was lower (6.8I). (G) CD34 staining shows a few of more mature microvessels $(\times 200)$. (H) VEGF expression is negative $(\times 400)$. 

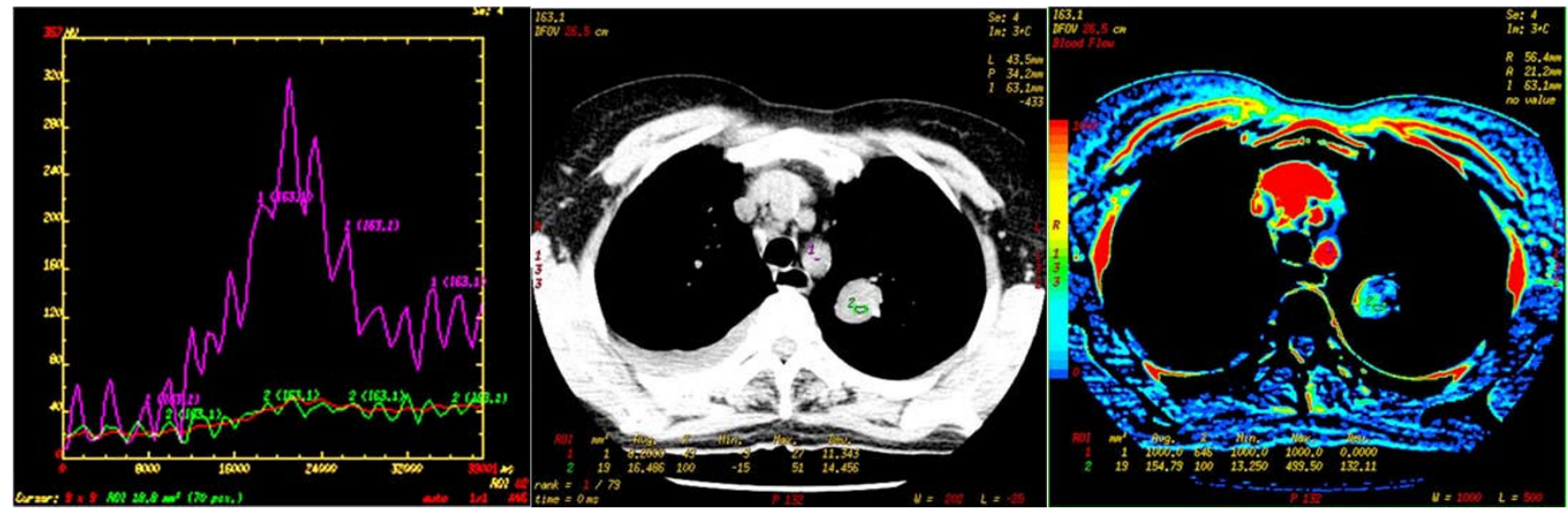

$\mathbf{A}$

$\mathbf{B}$

C

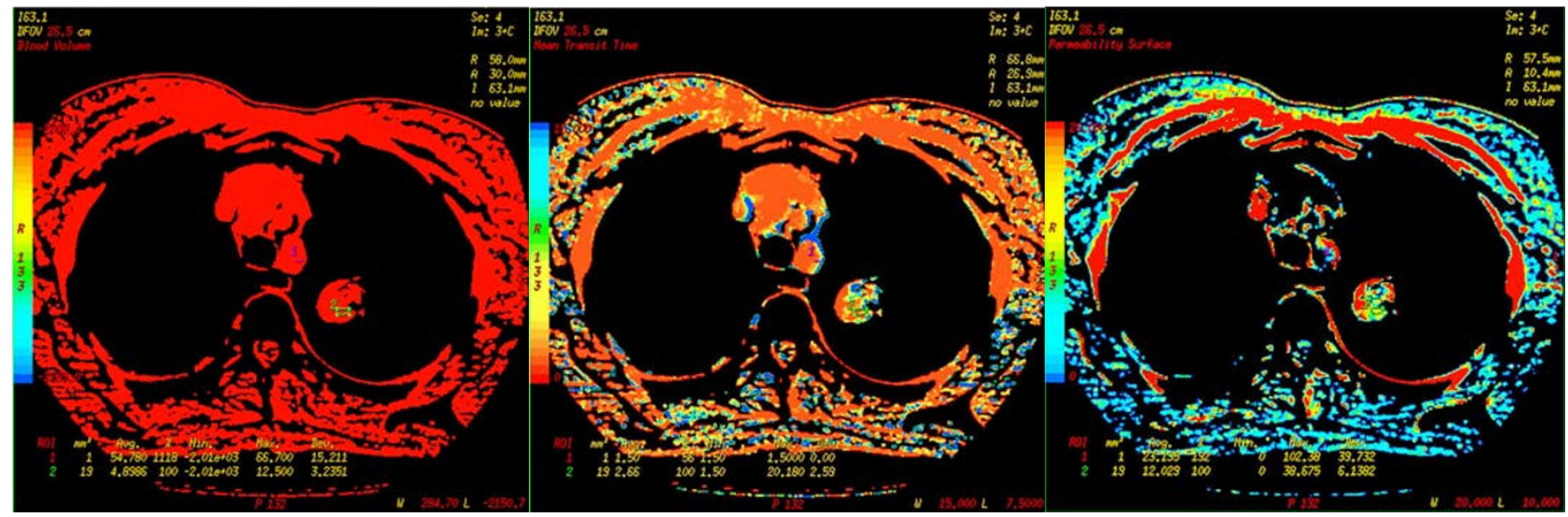

D

$\mathbf{E}$

F

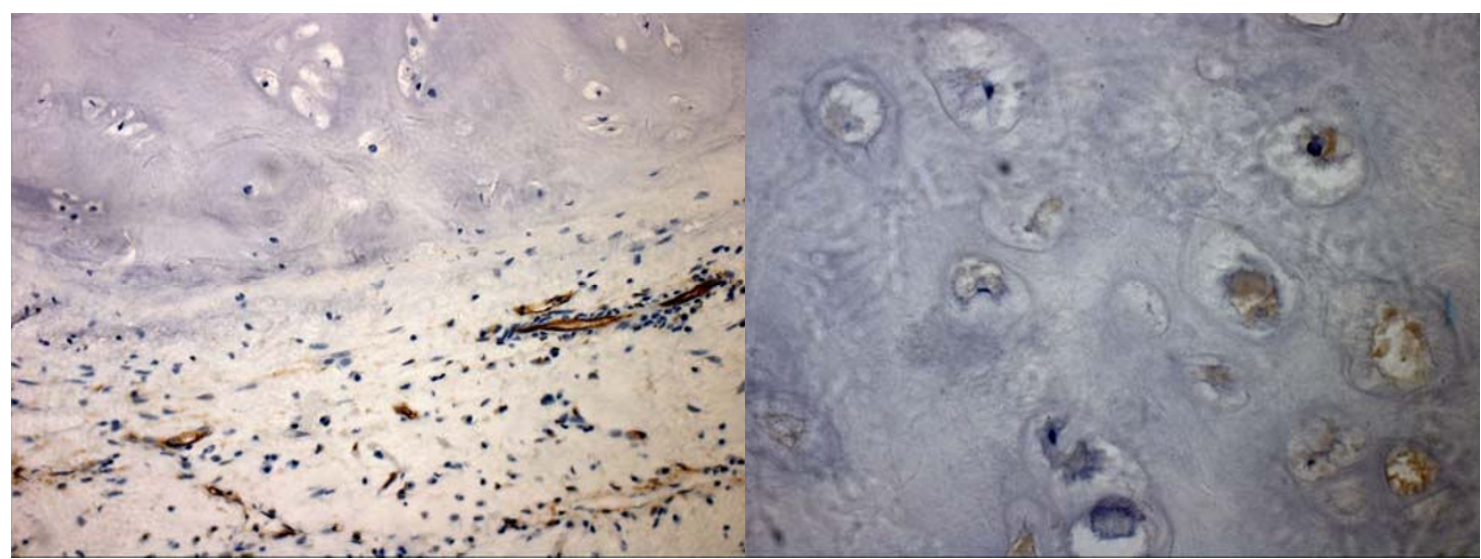

G

H

Figure 6

(A-H) Hamartoma found in apicoposterior segment of superior of the left lung of a 54-year-old male. (A) Time density curve. (B-F) (original image, BF, BV, MTT, PS) type I parametric maps, PS value was moderate (I2.029). (G) CD34 staining shows a few of more mature microvessels $(\times 200)$. (H) VEGF expression is negative $(\times 400)$. 
Table 3: Perfusion parameters and MVD of three groups of pulmonary nodules

\begin{tabular}{cccc}
\hline Parameter & $\begin{array}{c}\text { Peripheral lung cancer } \\
(\mathrm{n}=39)\end{array}$ & $\begin{array}{c}\text { Inflammatory nodule } \\
(\mathrm{n}=13)\end{array}$ & $\begin{array}{c}\text { Benign nodule } \\
(\mathrm{n}=12)\end{array}$ \\
\hline BV & $10.577 \pm 3.708$ & $8.542 \pm 2.581$ & $5.806 \pm 1.628$ \\
BF & $205.232 \pm 90.669$ & $160.902 \pm 68.412$ & $80.733 \pm 16.060$ \\
MTT & $7.472 \pm 3.760$ & $6.397 \pm 3.292$ & $5.760 \pm 2.884$ \\
PS & $19.703 \pm 7.660$ & $4.019 \pm 1.730$ & $3.797 \pm 2.186$ \\
PH & $60.790 \pm 8.336$ & $65.700 \pm 5.948$ & $27.833 \pm 9.072$ \\
PHpm/PHa (I00\%) & $20.611 \pm 3.243$ & $22.163 \pm 2.240$ & $8.893 \pm 2.624$ \\
MVD & $29.292 \pm 14.391$ & $33.966 \pm 15.548$ & $7.959 \pm 2.328$ \\
\hline
\end{tabular}

$*$ Data is the mean \pm SD.

Table 4: Comparison among the perfusion parameters and MVD of three groups of pulmonary nodules

\begin{tabular}{|c|c|c|c|c|c|c|c|}
\hline Parameter & $\begin{array}{l}\text { BV } \\
\text { (p) }\end{array}$ & $\begin{array}{l}\text { BF } \\
\text { (p) }\end{array}$ & $\begin{array}{c}\text { MTT } \\
(\mathrm{p})\end{array}$ & $\begin{array}{l}\text { PS } \\
\text { (p) }\end{array}$ & $\begin{array}{l}\mathrm{PH} \\
\text { (p) }\end{array}$ & $\begin{array}{l}\mathrm{PHpm} / \mathrm{PHa} \\
\text { (p) }\end{array}$ & $\begin{array}{l}\text { MVD } \\
\text { (p) }\end{array}$ \\
\hline Peripheral lung cancer $(n=39)$ and inflammatory nodule $(n=13)$ & 0.106 & 0.207 & 0.345 & 0.000 & 0.062 & 0.107 & 0.726 \\
\hline Peripheral lung cancer $(n=39)$ and benign nodule $(n=12)$ & 0.000 & 0.000 & 0.146 & 0.000 & 0.000 & 0.000 & 0.000 \\
\hline Inflammatory nodule $(n=13)$ and benign nodule $(n=12)$ & 0.013 & 0.003 & 0.653 & 0.990 & 0.000 & 0.000 & 0.000 \\
\hline
\end{tabular}

than those of peripheral lung cancer (all $\mathrm{P}<.05$ ), rather than BF, and MTT (all P > .05) (Tables 8, 9).

MVD, but not MTT, was positively correlated with $\mathrm{PH}$, $\mathrm{PHpm} / \mathrm{PHa}, \mathrm{BF}, \mathrm{BV}$, and PS of the peripheral lung cancer with VEGF positive expression. MVD was positively correlated with PS of the benign nodule with VEGF positive expression, rather than $\mathrm{PH}, \mathrm{PHpm} / \mathrm{PHa}, \mathrm{BF}, \mathrm{BV}$, and MTT. MVD was not correlated with PH, PHpm/PHa, BF, BV, PS, as well as MTT of the three groups of pulmonary nodules with VEGF negative expression (Table 10).

\section{Perfusion parametric maps of pulmonary nodules}

There were 3 types of CT perfusion parametric maps of pulmonary nodules. Type I was lack of central blood perfusion with sufficient peripheral blood perfusion (Figures 2C-2F, 5C-5F, 6C-6F); Type II was heterogeneity blood perfusion and much less blood perfusion in the area of necrosis (Figures 3C-3F); Type III was lack of central and peripheral blood perfusion (Figures 4C-4F).
There were 21 patients in type I cases (13 peripheral lung cancers, 2 inflammatory nodules, 4 tuberculoms, 2 hamartomas); 33 patients in type II cases (24 peripheral lung cancers, 9 inflammatory nodules) and 10 patients in type III cases ( 2 peripheral lung cancers, 2 inflammatory, 5 tuberculomas, 1 hamartoma). The perfusion pattern of peripheral lung cancer and inflammatory nodule was not statistically significant $(\mathrm{F}=2.559, \mathrm{P}=0.298)$. Both and benign nodule were statistically significant $(\mathrm{F}=19.984, \mathrm{P}$ $=0.000 ; \mathrm{F}=13.562, \mathrm{P}=0.001)$ (Table 11).

The size of the tumor in CT perfusion parametric maps was smaller than that of CT original images and there were more exudative process found in the nodules (Figures 4B$4 \mathrm{~F})$.

\section{Discussion}

A growing tumor needs added blood supply from adjacent tissues, because this is essentially required for tumor growth and metastatic spread. This process may be caused by the increasing release of angiogenic factors from the

Table 5: Comparison among the perfusion parameters and MVD of adenocarcinoma, squamous cell carcinoma, large and small cell carcinoma

\begin{tabular}{|c|c|c|c|c|c|c|c|c|}
\hline Parameter & Cases & BV & $\mathrm{BF}$ & MTT & PS & $\mathrm{PH}$ & $\mathrm{PHpm} / \mathrm{PHa}$ & MVD \\
\hline Adenocarcinoma & 24 & $10.173 \pm 3.624$ & $225.46 \pm 83.132$ & $6.673 \pm 3.821$ & $17.819 \pm 8.214$ & $62.329 \pm 8.084$ & $21.159 \pm 3.083$ & $27.483 \pm 14.85$ \\
\hline $\begin{array}{l}\text { Squamous cell } \\
\text { carcinoma }\end{array}$ & 11 & $10.244 \pm 3.528$ & $164.071 \pm 101.015$ & $8.876 \pm 3.371$ & $22.217 \pm 6.414$ & $56.436 \pm 8.456$ & $19.048 \pm 3.465$ & $30.828 \pm 15.559$ \\
\hline $\begin{array}{l}\text { Large and small } \\
\text { cell carcinoma }\end{array}$ & 4 & $13.92 \pm 3.925$ & $197.063 \pm 86.943$ & $8.413 \pm 3.969$ & $24.093 \pm 3.389$ & $63.525 \pm 6.52$ & $21.62 \pm 2.758$ & $35.918 \pm 5.683$ \\
\hline
\end{tabular}

*BV, BF, PS, MTT, PH, PHpm/PHa, and MVD among adenocarcinoma, squamous cell carcinoma, large and small cell carcinoma were not significantly (all P > 0.05). 
Table 6: Tumor differentiations of adenocarcinoma, squamous cell carcinoma, large and small cell carcinoma

\begin{tabular}{|c|c|c|c|c|c|c|c|c|c|}
\hline Parameter & Differentiation & Cases & BV & $\mathrm{BF}$ & MTT & PS & $\mathrm{PH}$ & $\mathrm{PHpm} / \mathrm{PHa}$ & MVD \\
\hline \multirow{3}{*}{$\begin{array}{l}\text { Adenocarcino } \\
\text { ma ( } 24 \text { cases) }\end{array}$} & well & 9 & $8.706 \pm 2.441$ & $205.723 \pm 77.469$ & $5.422 \pm 1.591$ & $15.949 \pm 5.736$ & $61.122 \pm 6.371$ & $20.718 \pm 3.001$ & $22.451 \pm 13.536$ \\
\hline & moderately & 10 & $9.261 \pm 3.448$ & $205.193 \pm 90.827$ & $8.755 \pm 5.0$ & $15.528 \pm 9.547$ & $59.95 \pm 6.435$ & $20.069 \pm 2.478$ & $22.831 \pm 12.514$ \\
\hline & poorly & 5 & $14.636 \pm 2.237$ & $301.518 \pm 6.290$ & $4.758 \pm 1.919$ & $25.766 \pm 4.142$ & $69.26 \pm 11.132$ & $24.134 \pm 2.889$ & $45.846 \pm 4.079$ \\
\hline \multirow{3}{*}{$\begin{array}{l}\text { Squamous cell } \\
\text { carcinoma } \\
\text { (I l cases) }\end{array}$} & well & 6 & $10.308 \pm 2.729$ & $182.63 \pm 105.873$ & 8.3624 .083 & $20.083 \pm 7.198$ & $59.717 \pm 5.138$ & $20.342 \pm 2.17$ & $28.902 \pm 16.223$ \\
\hline & moderately & 4 & $10.393 \pm 5.359$ & $157.448 \pm 110.379$ & $9.938 \pm 2.759$ & $22.993 \pm 3.081$ & $52.825 \pm 12.095$ & $17.933 \pm 4.852$ & $29.693 \pm 16.515$ \\
\hline & poorly & 1 & $9.26 \pm 0.0$ & $79.210 \pm 0.0$ & $7.710 \pm 0.0$ & $31.92 \pm 0.0$ & $51.20 \pm 0.0$ & $15.75 \pm 0.0$ & $46.93 \pm 0.0$ \\
\hline \multirow{3}{*}{$\begin{array}{l}\text { Large and } \\
\text { small cell } \\
\text { carcinoma } \\
\text { (4 cases) }\end{array}$} & well & 3 & $13.743 \pm 4.787$ & $165.083 \pm 72.129$ & $6.67 \pm 2.327$ & $23.003 \pm 3.179$ & $60.567 \pm 3.356$ & $20.46 \pm 1.828$ & $34.993 \pm 6.582$ \\
\hline & moderately & 0 & 0 & 0 & 0 & 0 & 0 & 0 & 0 \\
\hline & poorly & 1 & $14.45 \pm 0.0$ & $293.0 \pm 0.0$ & $13.64 \pm 0.0$ & $27.36 \pm 0.0$ & $72.4 \pm 0.0$ & $25.1 \pm 0.0$ & $38.69 \pm 0.0$ \\
\hline
\end{tabular}

tumor, such as vascular endothelial growth factor (VEGF), and subsequent increase in the extent of microvessel density (MVD) [11-14]. The resulting increase of MVD leads to increased perfusion and permeability of the blood capillaries. Owing to the increased MVD which is associated frequently with increased parameter values of a tumor at CT perfusion imaging [15], CT perfusion imaging can be interpreted as reflecting tumor vascularity. This discovery may aid in comparing the differentiation of benignancy and malignancy of the tumor $[16,17]$.

\section{Application value of TDC in the differentiation of benignancy and malignancy of the tumor}

We want to look into early changes in the TDC in a region of interest which correlates with blood flow per unit of tissue. It is found that sufficient vascular characterization may be completely provided in the first scans after an intravenous bolus of contrast medium. CT perfusion imaging should be carried out during an early and firstpass process of contrast medium enhancement. Therefore, the data acquisition time for scanning is crucial. This study indicated that more intact TDC of all the nodules appeared within 40 seconds, and 2-second duration time after an intravenous bolus of contrast medium was carried out.

The different appearances of TDC of pulmonary nodules mainly represented the differences in blood perfusion and diffusion [18]. This study indicated that TDC of peripheral lung cancer had a steeper slope, and there was an obvious increase of nodular HU value. This changed little after reaching peak. In fact, it became flat at the peak, and had a platform. The enhancement of peripheral lung cancer and aorta enhanced at an equal pace. However, the peak of TDC appeared slightly later than that of the aorta. This was in accordance to the reports of Littleton [19] and Han Mingjun [20] who proposed that most lung cancers had abundant vascular supply, which was from the bronchial arterial system. The blood perfusion of peripheral lung cancer is abundant, so, there was a steeper slope in TDC.

The interstitial space of peripheral lung cancer was found to be larger, and a near absence or substantial reduction of lymphatic flow was noted. The retarded flow found in intravascular and interstitial spaces in the washout phase from the interstitial space contribute to retention of contrast medium in peripheral lung cancer. Thus, TDC of pulmonary nodule hanged out a little after reaching the peak. It was found to be flat at peak, with a platform.

This study indicated that TDC of inflammatory nodule had a steeper slope, which declined immediately after reaching the peak. It also indicated that in most of the processes of pulmonary inflammatory nodules, the number and sizes of bronchial arteries and blood circum-

Table 7: Relationships between adenocarcinoma differentiations and parameters

\begin{tabular}{|c|c|c|c|c|c|c|c|}
\hline Parameter & $\begin{array}{l}\text { BV } \\
\text { (p) }\end{array}$ & $\begin{array}{l}\text { BF } \\
\text { (p) }\end{array}$ & $\begin{array}{l}\text { MTT } \\
(\mathrm{p})\end{array}$ & $\begin{array}{l}\text { PS } \\
\text { (p) }\end{array}$ & $\begin{array}{l}\mathrm{PH} \\
\text { (p) }\end{array}$ & $\begin{array}{c}\mathrm{PHpm} / \mathrm{PHa} \\
(\mathrm{p})\end{array}$ & $\begin{array}{l}\text { MVD } \\
\text { (p) }\end{array}$ \\
\hline Well $(n=9)$ and moderately $(n=10)$ & 0.679 & 1.000 & 0.198 & 0.999 & 0.738 & 0.615 & 0.945 \\
\hline Well $(n=9)$ and poorly $(n=5)$ & 0.001 & 0.018 & 0.897 & 0.011 & 0.066 & 0.038 & 0.002 \\
\hline Poorly $(n=5)$ and moderately $(n=10)$ & 0.003 & 0.025 & 0.130 & 0.038 & 0.035 & 0.014 & 0.002 \\
\hline
\end{tabular}

*Because there are fewer cases of squamous cell carcinoma, large and small cell carcinoma, relationships between their differentiations and parameters were not compared. In the case of adenocarcinoma, BV, BF, PS, PHpm/PHa, and MVD between poorly and well differentiation and between poorly and moderately differentiation were statistically significant (all $\mathrm{P}<0.05$ ). 
Table 8: Comparison among the perfusion parameters and MVD between VEGF positive and negative expression of three groups of peripheral pulmonary nodules

\begin{tabular}{|c|c|c|c|c|c|c|c|c|}
\hline $\begin{array}{l}\text { Pulmonary } \\
\text { nodule }\end{array}$ & Parameter & BV & $\mathrm{BF}$ & MTT & PS & $\mathrm{PH}$ & $\begin{array}{l}\mathrm{PHpm} / \mathrm{PHa} \\
(100 \%)\end{array}$ & MVD \\
\hline \multirow[t]{4}{*}{$\begin{array}{l}\text { Peripheral } \\
\text { lung cancer }\end{array}$} & $\begin{array}{c}\text { VEGF positive } \\
\text { expression }(n=29)\end{array}$ & $\mathrm{I} I .457 \pm 3.593$ & $225.406 \pm 83.556$ & $7.326 \pm 3.807$ & $21.638 \pm 7.050$ & $62.531 \pm 8.579$ & $21.285 \pm 3.238$ & $32.495 \pm|4.54|$ \\
\hline & $\begin{array}{l}\text { VEGF negative } \\
\text { expression }(n=10)\end{array}$ & $8.025 \pm 2.859$ & $146.728 \pm 88.744$ & $7.896 \pm 3.787$ & $14.090 \pm 6.785$ & $55.740 \pm 5.132$ & $18.657 \pm 2.466$ & $20.002 \pm 9.363$ \\
\hline & $\mathrm{F}$ & 7.449 & 6.394 & 0.167 & 8.680 & 5.522 & 5.455 & 6.400 \\
\hline & $P$ & 0.010 & 0.016 & 0.685 & 0.006 & 0.024 & 0.025 & 0.016 \\
\hline \multirow[t]{2}{*}{$\begin{array}{l}\text { Inflammatory } \\
\text { nodule }\end{array}$} & $\begin{array}{c}\text { VEGF positive } \\
\text { expression }(n=0)\end{array}$ & I & I & I & I & I & 1 & I \\
\hline & $\begin{array}{c}\text { VEGF negative } \\
\text { expression }(n=13)\end{array}$ & $8.542 \pm 2.581$ & $160.902 \pm 68.412$ & $6.397 \pm 3.292$ & $4.019 \pm 1.730$ & $65.700 \pm 5.948$ & $22.163 \pm 2.240$ & $33.966 \pm 15.548$ \\
\hline \multirow[t]{4}{*}{$\begin{array}{l}\text { Benign } \\
\text { nodule }\end{array}$} & $\begin{array}{l}\text { VEGF positive } \\
\text { expression }(n=3)\end{array}$ & $6.620 \pm 2.231$ & $83.070 \pm 25.153$ & $6.320 \pm 1.890$ & $5.047 \pm 2.945$ & $30.733 \pm 14.199$ & $9.793 \pm 4.177$ & $8.877 \pm 3.285$ \\
\hline & $\begin{array}{l}\text { VEGF negative } \\
\text { expression }(n=9)\end{array}$ & $5.534 \pm 1.439$ & $79.954 \pm 13.919$ & $5.573 \pm 3.222$ & $3.380 \pm 1.903$ & $26.867 \pm 7.652$ & $8.592 \pm 2.168$ & $7.653 \pm 2.081$ \\
\hline & $\mathrm{F}$ & 1.000 & 0.078 & 0.139 & 1.350 & 0.386 & 0.448 & 0.599 \\
\hline & $P$ & $0.34 I$ & 0.786 & 0.717 & 0.272 & 0.548 & 0.519 & 0.457 \\
\hline
\end{tabular}

*Data is the mean $\pm S D$.

fluence of bronchial veins had increased. Moreover, diffused thrombosis at the arterioles of the pulmonary circulation was observed and the microvessel structure was found to be made up of coarctate capillary network from the bronchial artery [19]. Moreover, it was found that the lung has two systems-bronchial artery and pulmonary artery of blood supply. When inflammatory nodules, ratio of ventilation and blood flow changed, the pulmonary arteries became narrow or occlusive, and bronchial artery became expanded. The vascular supply was actually found to be from the bronchial arteries. Thus, blood perfusion of the inflammatory nodule is abundant, and has a steeper slope in TDC. Flow of contrast medium through the intravascular space occurred through relatively straight vessels with a normal configuration. There was an accelerated, active lymphatic flow, and diffusion of contrast medium increased. Thus, TDC of inflammatory nodule declined earlier than that of peripheral lung cancer after reaching the peak.

This study indicated that TDC of benign nodule (tuberculoma and hamartoma) manifested as a flat trend. There was no a steeper slope, or an obvious increase in nodular material and hamartoma was mainly from cartilage and fibrous tissues. Therefore, MVD is smaller, and vascular supply of tuberculoma and hamartoma is not abundant. There is lesser blood circumfluence in vessels and extravascular space. This resulted in a faster diffusion in tuberculoma and hamartoma. Thus, TDC of benign nodule manifested in a flat trend. Tthere was no steeper slope, or increase in nodular CT value.

This study indicated that TDC may depict a hemadynamic character of the pulmonary nodules. These characterizations may act as reference indexes for differentiating benign and malignant pulmonary nodules. TDC of peripheral lung cancer was similar to that of inflammatory nodule. Both TDC were shown to be obviously different from that of benign nodule. This coincided with the study of Zhang et al. [21]. However, Zhang et al. [21] believed that after inflammatory nodules reached maximum enhancement, the TDC declined and then rose again. This did not coincide with our study for two reasons. Firstly, contrast medium distributed in the vessels rather than in extravascular space during data acquisition of 40-seconds. After an intravenous bolus of conventional iodinated con-

Table 9: Comparison among the perfusion parameters and MVD between VEGF positive and negative expression of three groups of peripheral pulmonary nodules

\begin{tabular}{|c|c|c|c|c|c|c|c|c|c|}
\hline & Parameter & & BV & $\mathrm{BF}$ & MTT & PS & $\mathrm{PH}$ & $\mathrm{PHpm} / \mathrm{PHa}$ & MVD \\
\hline $\begin{array}{l}\text { VEGF positive } \\
\text { expression }\end{array}$ & Peripheral lung cancer $(n=29)$ and benign nodule $(n=3)$ & $P$ & 0.031 & 0.007 & 0.658 & 0.000 & 0.000 & 0.000 & 0.010 \\
\hline \multirow{3}{*}{$\begin{array}{l}\text { VEGF negative } \\
\text { expression }\end{array}$} & Peripheral lung cancer $(n=10)$ and inflammatory nod $(n=13)$ & $P$ & 0.616 & 0.968 & 0.308 & 0.003 & 0.000 & 0.001 & 0.043 \\
\hline & Peripheral lung cancer $(n=10)$ and benign nodule $(n=9)$ & $P$ & 0.033 & 0.114 & 0.152 & 0.002 & 0.000 & 0.000 & 0.007 \\
\hline & Inflammatory nodule $(n=13)$ and benign nodule $(n=9)$ & $\mathrm{P}$ & 0.008 & 0.003 & 0.585 & 0.818 & 0.000 & 0.000 & 0.000 \\
\hline
\end{tabular}

CT value. Tuberculoma occurred mainly from caseous

trast material, the TDC reflected sufficient vascular charac- 
Table 10: Correlation between MVD and perfusion parameters of three groups of peripheral pulmonary nodules with VEGF positive and negative expression

\begin{tabular}{|c|c|c|c|c|c|}
\hline \multirow[t]{2}{*}{ Pulmonary nodule } & \multirow[t]{2}{*}{ Parameter } & \multicolumn{2}{|c|}{ VEGF positive expression } & \multicolumn{2}{|c|}{ VEGF negative expression } \\
\hline & & $r$ & $P$ & $r$ & $P$ \\
\hline \multirow[t]{6}{*}{ Peripheral lung cancer } & BV & 0.429 & 0.020 & 0.106 & 0.771 \\
\hline & BF & 0.432 & 0.019 & 0.013 & 0.971 \\
\hline & MTT & -0.218 & 0.256 & -0.174 & 0.630 \\
\hline & PS & 0.512 & 0.004 & 0.114 & 0.754 \\
\hline & $\mathrm{PH}$ & 0.444 & 0.016 & 0.533 & 0.112 \\
\hline & $\mathrm{PHpm} / \mathrm{PHa}$ & 0.415 & 0.025 & 0.452 & 0.190 \\
\hline \multirow[t]{6}{*}{ Inflammatory nodule } & BV & 1 & 1 & 0.135 & 0.661 \\
\hline & $\mathrm{BF}$ & I & I & 0.104 & 0.734 \\
\hline & MTT & I & I & -0.252 & 0.407 \\
\hline & PS & I & I & 0.299 & 0.321 \\
\hline & $\mathrm{PH}$ & I & I & 0.067 & 0.829 \\
\hline & $\mathrm{PHpm} / \mathrm{PHa}$ & I & I & 0.344 & 0.250 \\
\hline \multirow[t]{6}{*}{ Benign nodule } & BV & 0.957 & 0.188 & 0.407 & 0.277 \\
\hline & $\mathrm{BF}$ & 0.996 & 0.055 & 0.360 & 0.341 \\
\hline & MTT & -0.992 & 0.083 & -0.544 & 0.130 \\
\hline & PS & 0.999 & 0.026 & 0.535 & 0.138 \\
\hline & $\mathrm{PH}$ & 0.991 & 0.086 & $0.44 I$ & 0.235 \\
\hline & $\mathrm{PHpm} / \mathrm{PHa}$ & 0.983 & 0.116 & 0.468 & 0.203 \\
\hline
\end{tabular}

terization but did not reflect TDC of extravascular characterization in this study. Secondly, this discovery correlated with the scanner mode and application of the 16-slice spiral CT machine in this study.

TDC of benign nodule was obviously different from that of peripheral lung cancer and inflammatory nodule. When TDC manifested as a flat trend, there is neither a steeper slope nor obvious increase of nodular HU value. Therefore, morphologic characteristics and patient history may be used, and tuberculoma or hamartoma may be considered first before other pulmonary lesions are proved.

Table I I: Type of perfusion parametric maps showing three groups of pulmonary nodules

\begin{tabular}{lccc}
\hline & Typel & Typell & Type III \\
\hline Peripheral lung cancer $(\mathrm{n}=39)$ & 13 & 24 & 2 \\
Inflammatory nodule $(\mathrm{n}=13)$ & 2 & 9 & 2 \\
Benign nodule $(\mathrm{n}=12)$ & 6 & 0 & 6
\end{tabular}

*The perfusion patterns of peripheral lung cancer and inflammatory nodule were not statistically significant $(F=2.559, p=0.298)$, but both and benign nodule were statistically significant $(F=19.984, P=$ $0.000 ; F=13.562, P=0.001)$.

\section{Clinical application value of perfusion parameters in the} differentiation of benignancy and malignancy of tumor

This study indicated that $\mathrm{PH}, \mathrm{PHpm} / \mathrm{PHa}, \mathrm{BF}, \mathrm{BV}$ value of peripheral lung cancer and inflammatory nodule were not statistically significant. Both showed significantly higher PH, PHpm/PHa, BF, BV value than those of benign nodule. Peripheral lung cancer showed significantly higher PS value than that of inflammatory nodule and benign nodule. PS value of inflammatory nodule and benign nodule were not statistically significant. MTT value was not statistically significant among the three groups of nodules. In the case of adenocarcinoma, BV, BF, PS, PHpm/PHa between poorly and well differentiation and between poorly and moderately differentiation were statistically significant.

The reason may be owing to the fact that the vascular supply of malignant nodule and inflammatory nodule is more abundant, and therefore, both blood perfusion have increased. The vascular supply of benign nodule is lesser, therefore, its blood perfusion is obviously less than that of malignant nodule and inflammatory nodule. This coincided with the study results of Swensen et al. [5,7,22,23] and Zhang et al. [21], who compared PH, PHpm/PHa or perfusion value of malignant, inflammatory, and benign nodule, and suggested that malignant and inflammatory nodule showed higher $\mathrm{PH}, \mathrm{PHpm} / \mathrm{PHa}$ or higher perfusion value than those of benign nodule. 
The result of this study indicated that there was a better consistency among BF, BV, PS, PH, PHpm/PHa in peripheral lung cancer, inflammatory nodule, and benign nodule cases. This result suggested that the five perfusion parameters may better reflect blood perfusion status, malignant and benign degree of pulmonary nodules.

PS value reflects one-way transmission speed for contrast material to enter cell spaces by capillary endothelium, and it reflects the diffusion coefficient of blood vessel endothelial interspace of tumor angiogenesis. There were straighter branches of blood vessels, mature blood capillaries, and near normal permeability surfaces of blood capillaries in benign and inflammatory nodules. Therefore, PS value of inflammatory nodule and benign nodule was lower, and not statistically significant. For peripheral lung cancer, there is found to have more developmental immaturity of tumor capillary, no intact vessel wall, and increased permeability surface of blood capillary which resulted from VEGF and other vascular growth factors. Therefore, the PS value of peripheral lung cancer is higher.

$\mathrm{PH}, \mathrm{PHpm} / \mathrm{PHa}, \mathrm{BF}, \mathrm{BV}$ value of peripheral lung cancer and inflammatory nodule were usually significantly higher than those of benign nodule. Peripheral lung cancer usually showed significantly higher PS value than that of inflammatory nodule and benign nodule. when a pulmonary nodule shows that $\mathrm{PH}, \mathrm{PHpm} / \mathrm{PHa}, \mathrm{BV}$ and $\mathrm{BF}$ are higher, PS is usually higher. It is possible that before other pulmonary nodules are found, peripheral lung cancer may be considered first. When pulmonary nodules show PH, PHpm/PHa, BV, BF are higher, PS is found to be lower, and inflammatory nodule may be considered first.

\section{Application value of perfusion parametric maps of pulmonary nodules}

The findings of our study indicated that perfusion map in type I usually showed up in tuberculoma and hamartoma, and there was shown to be a lack of central blood perfusion, and sufficient peripheral blood perfusion. Perfusion map in type II usually showed up in inflammatory nodule and peripheral lung cancer. In this case, there was the presence of heterogeneity blood perfusion, and area of necrosis was found to be less. Perfusion map in type III usually also showed up in tuberculoma, hamartoma and inflammatory nodule, and there was found to be a lack of central and peripheral blood perfusion.

In perfusion parametric map of pulmonary nodule, there is found to be a lack of central blood perfusion and sufficient peripheral blood perfusion before other pulmonary lesions are proved. Morphologic characteristics and patient history may be used, and benign pulmonary nodule (tuberculoma or hamartoma) may be considered first. However, other pulmonary nodules may found not to completely excluded, because some adenocarcinomas may be filled with mucus. Also fewer inflammatory nodules may be filled with pus, and have lack of central perfusion.

This study indicated that the low-perfusion area of necrosis and mucus or cavitation area could diminish the mean perfusion parameter values. Therefore, when drawing up region of interest, it is considered that pulmonary nodule, low perfusion and cavitation should be avoided as far as possible.

The size of tumor in CT perfusion parametric maps was usually smaller than that of original images when there were more exudative process shown in tumors. Therefore, CT perfusion parametric maps displayed by color gray scale may reveal the essence of pulmonary nodules and differences in regional blood perfusion status more directly, clearly, realistically, and reliably. This will probably aid in paracentesis and biopsy of the essence of pulmonary nodule by CT guidance.

\section{Relationship between perfusion parameters and MVD and VEGF in pulmonary nodules}

Angiogenesis is a complicated process, as it is controlled by vascular growth factors and vascular growth inhibitor factors $[24,25]$, This is because the endothelial cells which have resided in blood capillary and postcapillary vein will enter again itto cell generation cycle. As a result, there will be degrading basal membrane under endothelia cells and this will form blood capillary spore. In turn, blood capillary will move towards peripheral tissues by a pullulate way. Thus, when a tumor has no passage to directly contact with the eutaxia circulation system, it must induce the vicinity vessels to erupt the new vessels.

Growth of tumor will suffer limitation if there is occurrence of no angiogenesis. Tumor cells obtain nutrition only when there is diffusion. Tumor will stop growing after achieving 1-2 nm diameter or thickness of about $10^{7}$ cells if there is not enough oxygen and nutrition supply. However, growth of tumor will accelerate once new vessels are put into tumor tissues, and the nutrition supply of tumor transforms perfusion from diffusion process. Therefore, the tumor is a growth that typically depends on supply from the blood vessels. Although there are many kinds of vascular growth factors, VEGF played a crucial role in tumor angiogenesis because of its speciality factor in promoting tumor karyokinesis [25]. MVD is the number of microvessel per unit area which is counted by using some specific antibodies (for instance CD34 monoclonal antibody)to mark microvessel endothelial cells of tumor tissues. 
This study indicated that in the case of VEGF positive expression, MVD was found to be positively correlated with $\mathrm{PH}, \mathrm{PHpm} / \mathrm{PHa}, \mathrm{BF}, \mathrm{BV}$, and PS of peripheral lung cancer. This coincided with the studies of Yi et al. [26], Tateishi et al. [27] and Li Shenjiang et al. [8]. Yi et al. and Tateishi et al. described that a significantly positive correlation was found between enhancement of MVD and/or VEGF of malignant pulmonary nodule. Li Shenjiang et al [8]. reported that enhancement and perfusion value were significantly and positively correlated with MVD and VEGF of malignant pulmonary nodule, and MTT value was not significantly correlated with MVD and VEGF.

This study also indicated that VEGF expression of 32 cases (29 peripheral lung cancer, 0 inflammatory nodule, 3 benign nodule) were positive. MVD was not correlated with $\mathrm{PH}, \mathrm{PHpm} / \mathrm{PHa}, \mathrm{BF}, \mathrm{BV}$, and PS of three groups of pulmonary nodules with VEGF negative expression. The peripheral lung cancers with VEGF positive expression showed significantly higher $\mathrm{PH}, \mathrm{PHpm} / \mathrm{PHa}, \mathrm{BF}, \mathrm{BV}$, and $P S$ value than those of the peripheral lung cancers with VEGF negative expression, and those of benign nodule with VEGF positive expression.

It is clear that $\mathrm{PH}, \mathrm{PHpm} / \mathrm{PHa}, \mathrm{BF}, \mathrm{BV}$, and PS value can reflect MVD measurement and VEGF expression of benign and malignant pulmonary nodules. This explains that $\mathrm{PH}$, $\mathrm{PHa} / \mathrm{PHpm}, \mathrm{BF}, \mathrm{BV}$, PS may act as angiogenesis index of pulmonary nodules, and have potential value of studied pulmonary nodule angiogenesis.

\section{Conclusion}

16-slice spiral CT perfusion imaging closely correlated with tumor angiogenesis and reflected MVD measurement and VEGF expression. It provided not only a noninvasive method of quantitative assessment for blood flow patterns of peripheral pulmonary nodules.

\section{Abbreviations}

VEGF: Vascular endothelial growth factor; TDC: Time density curve; MVD: Microvessel density; PH: Peak height; PHpm/PHa: Peak height ratio of pulmonary nodule to aorta; BF: Blood flow; BV: Blood volume; PS: Permeability surface; MTT: Mean transit time.

\section{Competing interests}

The authors declare that they have no competing interests.

\section{Authors' contributions}

SHM created the original study design, wrote the protocol, and extracted the data. HBL, BHJ and ZXW read an earlier version of the manuscript and gave insightful comments and suggestions. SHM, HBL and XLC undertook CT scanning and analyses of CT images. SHM, HBL and WM inquired about cases and undertook immunohistochemi- cal staining. ZXW and ZGH did statistical testings. All authors contributed to both protocol and final reports.

\section{Acknowledgements}

This study was supported by grants from the Nature Science Foundation of Guangdong Province of China (0630 I077), the Traditional Chinese Medicine Administration Foundation of Guangdong Province of China (2060134), the Health Department Foundation of Guangdong Province of China (A200742I), the Shantou Technology Bureau Science Foundation of China (Shantou government technology (2006)85), and National Nature Science Foundation of China (30700235).

The authors are grateful to Zu-De Zhu, Tao Huang for excellent technical assistance; Li-Hua Sun for immunohistochemistry experiment; Bao-Jun Huang for conducting the mass analyses of CT perfusion images, as well as the English editorial assistance of John K. Lim, M.D., from USA, professor Grace from Singapore, Junqing Cui, PH.D. from USA, are gratefully acknowledged.

\section{References}

I. Klingenbeck-Regn K, Schaller S, Flohr T, Ohnesorge B, Kopp AF, Baum U: Subsecond multi slice computed tomography: basics and applications. Eur J Radiol 1999, 31: I I0-124.

2. Vaupel P, Kallinowski F, Okunieff P: Blood flow, oxygen and nutrient supply, and metabolic microenvironment of human tumors: a review. Cancer Res 1989, 49:6449-6465.

3. Miles KA: Tumour angiogenesis and its relation to contrast enhancement on computed tomography: a review. Eur J Radiol 1999, 30: 198-205.

4. Schaefer JF, Vollmar J, Schick F, Vonthein R, Seemann MD, Aebert H, Dierkesmann R, Friedel G, Claussen CD: Solitary pulmonary nodules: dynamic contrast-enhanced MR imaging-perfusion differences in malignant and benign lesions. Radiology 2004, 232:544-553.

5. Swensen SJ, Brown LR, Colby TV, Weaver AL, Midthun DE: Lung nodule enhancement at CT: prospective findings. Radiology 1996, 201:447-455.

6. Ohno Y, Hatabu H, Takenaka D, Adachi S, Kono M, Suqimura K: Solitary pulmonary nodules: potential role of dynamic MR imaging in management initial experience. Radiology 2002, 224:503-5II.

7. Swensen S], Viggiano RW, Midthun DE, Müller NL, Sherrick A, Yamashita K, Naidich DP, Patz EF, Hartman TE, Muhm JR, Weaver AL: Lung nodule enhancement at CT: multicenter study. Radiology 2000, 2 1 4:73-80

8. Li Shen-jiang, Xiao Xiang-sheng, Liu Shi-yuan: Blood flow patterns of solitary pulmonary nodules with enhancement: clinical value of multislice spiral CT. Chin J Radiol 2004, 38: I062-1068.

9. Mattern J, Koomagi R, Volm M: Association of vascular endothelial growth factor expression with intratumoral microvessel density and tumor cell proliferation in human epidermoid lung carcinoma. BrJ Cancer 1996, 73:931-934.

10. Weidner N: Tumor vascularity and proliferation: Clear evidence of a close relationship. J Pathol 1999, 189:297-299.

II. Weidner N, Semple JP, Welch WR, Folkman J: Tumor angiogenesis and metastasis correlation in invasive breast carcinoma. N EnglJ Med I99I, 324:I-8.

12. Fontanini G, Bigini D, Vignati S, Basolo F, Mussi A, Lucchi M, Chine S, Angeletti CA, Harris AL, Bevilacqua G: Microvessel count predicts metastatic disease and survival in non-small cell lung cancer. J Pathol 1995, 177:57-63.

13. Fontanini G, Vignati S, Boldrini L, Chinè S, Silvestri V, Lucchi M, Mussi $A$, Angeletti CA, Bevilacqua G: Vascular endothelial growth factor is associated with neovascularization and influences progression of non-small cell lung carcinoma. Clin Cancer Res 1997, 3:86I-865.

14. Shibusa T, Shijubo N, Abe S: Tumor angiogenesis and vascular endothelial growth factor expression in stage I lung adenocarcinoma. Clin Cancer Res 1998, 4: I 483-I487.

15. Ahn MJ, Jang SJ, Park YW, Choi JH, Oh HS, Lee CB, Paik HK, Park CK: Clinical prognostic values of vascular endothelial growth fac- 
tor, microvessel density, and P53 expression in esophageal carcinomas. J Korean Med Sci 2002, 17:20I-207.

16. Tateishi U, Nishihara H, Watanabe S, Morikawa T, Abe K, Miyasaka $\mathrm{K}$ : Tumour angiogenesis and dynamic $\mathrm{CT}$ in lung adenocarcinoma: radiologic-pathologic correlation. J Comput Assist Tomogr 200I, 25:23-27.

17. Fujimoto K, Abe T, Müller NL, Terasaki H, Kato S, Sadohara J, Kono R, Edamitsu O, Ishitake T, Hayashi A, Rikimaru T, Hayabuchi N: Small peripheral pulmonary carcinomas evaluated with dynamic MR imaging: correlation with tumor vascularity and prognosis. Radiology 2003, 227:786-793.

18. Zhang Minming, Zhou Hua, Zhou Yu: Evaluation of pulmonary carcinoma angiogenesis by dynamic contrast-enhanced $\mathrm{CT}$ and MR functional imaging. Radiol Practice 2005, 20:286-290.

19. Littleton JT, Durizch ML, Moeller G, Herbert DE: Pulmonary masses: contrast enhancement. Radiology 1990, 177:86I-87|.

20. Han Mingjun, Feng Gansheng, Yang Jianyong, Su Hong-ying, Zhao Zhong-chun: The pulmonary artery doesn't participate in the blood supply of lung cancer:experimental and DSA study. Chin J Radiol 2000, 34:802-804.

21. Zhang M, Kono M: Solitary pulmonary nodules: evaluation of blood flow patterns with dynamic CT. Radiology 1997, 205:47|-478.

22. Swensen SJ, Browen LR, Colby TV, Weaver AL: Pulmonary nodules: CT evaluation of enhancement with iodinated contrast material. Radiology 1995, 194:393-398.

23. Swensen SJ, Morin RL, Schueler BA, Brown LR, Cortese DA, Pairolero PC, Brutinel WM: Solitary pulmonary nodule: $C T$ evaluation of enhancement with iodinated contrast material-a preliminary report. Radiology 1992, 1 82:343-347.

24. Miles KA, Griffiths MR, Fuentes MA: Standardized perfusion value: universal $C T$ contrast enhancement scale that correlates with FDG PET in lung nodules. Radiology 200I, 220:548-553.

25. O'Byrne KJ, Koukourakis MI, Giatromanolaki A, Cox G, Turley H, Steward WP, Gatter K, Harris AL: Vascular endothelial growth factor, platelet-derived endothelial cell growth factor and angiogenesis in non-small-cell lung cancer. $\mathrm{Br} J$ Cancer 2000, 82: $1427-1432$.

26. Yi CA, Lee KS, Kim EA, Han J, Kim H, Kwon OJ, Jeong YJ, Kim S: Solitary pulmonary nodules: Dynamic enhanced multi-detector row CT study and comparison with vascular endothelial growth factor and microvessel density. Radiology 2004, 233:191-199.

27. Tateishi U, Kusumoto M, Nishihara H, Nagashima K, Morikawa T, Moriyama N: Contrast-enhanced dynamic computed tomography for the evaluation of tumor angiogenesis in patients with lung carcinoma. Cancer 2002, 95:835-842.

\section{Pre-publication history}

The pre-publication history for this paper can be accessed here:

http://www.biomedcentral.com/1471-2407/8/186/pre pub
Publish with BioMed Central and every scientist can read your work free of charge

"BioMed Central will be the most significant development for disseminating the results of biomedical research in our lifetime. "

Sir Paul Nurse, Cancer Research UK

Your research papers will be:

- available free of charge to the entire biomedical community

- peer reviewed and published immediately upon acceptance

- cited in PubMed and archived on PubMed Central

- yours - you keep the copyright
BioMedcentral 